\title{
SPECTRAL PROPERTIES AND RIGIDITY FOR SELF-EXPANDING SOLUTIONS OF THE MEAN CURVATURE FLOWS
}

\author{
XU CHENG AND DETANG ZHOU
}

\begin{abstract}
In this paper, we study self-expanders for mean curvature flows. First we show the discreteness of the spectrum of the drifted Laplacian on them. Next we give a universal lower bound of the bottom of the spectrum of the drifted Laplacian and prove that this lower bound is achieved if and only if the self-expander is the Euclidian subspace through the origin. Further, for self-expanders of codimension 1, we prove an inequality between the bottom of the spectrum of the drifted Laplacian and the bottom of the spectrum of weighted stability operator and that the hyperplane through the origin is the unique self-expander where the equality holds. Also we prove the uniqueness of hyperplane through the origin for mean convex self-expanders under some condition on the square of the norm of the second fundamental form.
\end{abstract}

\section{INTRODUCTION}

In this paper we study self-expanding solutions for the mean curvature flow (MCF), i.e. self-expanders. Recall that an $n$-dimensional smooth selfexpander $\Sigma^{n}$ is a submanifold immersed in the Euclidean space $\left(\mathbb{R}^{n+k}, g_{0}\right)$, $k \geq 1$, satisfying

$$
\mathbf{H}=\frac{1}{2} x^{\perp},
$$

where $x$ denotes the position vector in $\mathbb{R}^{n+k}, \perp$ denotes the orthogonal projection onto the normal bundle of $\Sigma$, and $\mathbf{H}$ is the mean curvature vector of $\Sigma$ at $x$.

In the case of codimension $1,(1.1)$ is equivalent to that the mean curvature $H$ satisfies

$$
H=-\frac{1}{2}\langle x, \mathbf{n}\rangle
$$

where $\mathbf{n}$ denotes the unit normal field of $\Sigma$.

Equivalently, $\Sigma$ is a self-expander if and only if $\sqrt{t} \Sigma, t \in(0, \infty)$ is a mean curvature flow.

Self-expanders are very important in the study of MCF. They describe both the asymptotic longtime behavior for MCF and the local structure

2000 Mathematics Subject Classification. Primary: 58C42; Secondary: 58E30.

The authors are partially supported by CNPq and Faperj of Brazil. 
of MCF after the singularities in the very short time. See the works by Ecker and Huisken [12] and Stavrou [20]. Self-expanders also arise as the mean curvature evolution of cones. In Lecture 2 of [17], Ilmanen studied the existence of E-minimizing self-expanding hypersurfaces which converge to prescribed closed cones at infinity in Euclidean space. It is known that the singular minimal cones are the singular self-expanders. Recently, Ding 10 obtained some results on minimal cones and self-expanders. There are other works in self-expanders (see, e.g. [1, 13], 20] and references therein).

It is well known that a self-expander $\Sigma$ is a critical point of the weighted volume functional with weighted volume element $e^{\frac{|x|^{2}}{4}} d \sigma$, where $d \sigma$ is the volume element of $\Sigma$. On the other hand it can be viewed as a minimal submanifold under the conformal metric $e^{\frac{|x|^{2}}{2 n}} g_{0}$ on $\mathbb{R}^{n+k}$ (see more details, e.g. [3, 4]).

On a self-expander, an important operator is the drifted Laplacian $\mathscr{L}=$ $\Delta+\frac{1}{2}\langle x, \nabla \cdot\rangle$. The reason is that $\mathscr{L}$ is a densely defined self-adjoint operator in the weighted $L^{2}$ space $L^{2}\left(\Sigma, e^{\frac{|x|^{2}}{4}} d \sigma\right)$ of square integrable functions.

We discuss the spectral property of the operator $\mathscr{L}$ and show the discreteness of the spectrum of $\mathscr{L}$. More precisely,

Theorem 1.1. For a complete $n$-dimensional properly immersed self-expander $\Sigma$ in $\mathbb{R}^{n+k}, k \geq 1$, the spectrum of the drifted Laplacian $\mathscr{L}=\Delta+\frac{1}{2}\langle x, \nabla \cdot\rangle$ on $\Sigma$ is discrete. In particular, the bottom $\lambda_{1}$ of the spectrum of $\mathscr{L}$ is the first weighted $L^{2}$ eigenvalue of $\mathscr{L}$.

The discreteness of the spectrum of $\mathscr{L}$ means that the embeding of the Sobolev space $W_{0}^{1,2}\left(\Sigma, e^{\frac{|x|^{2}}{4}} d \sigma\right)$ into $L^{2}\left(\Sigma, e^{\frac{|x|^{2}}{4}} d \sigma\right)$ is compact. It is worthy of mentioning that self-shrinkers for MCF also have the same property. In [5], we showed that for a complete $n$-dimensional properly immersed selfshrinker in $\mathbb{R}^{n+k}$, the spectrum of its corresponding drifted Laplacian $\mathcal{L}=$ $\Delta-\frac{1}{2}\langle x, \nabla \cdot\rangle$ is discrete.

For a complete properly immersed self-shrinker, 0 is obviously the bottom of the spectrum of the operator $\mathcal{L}$ since the properness, polynomial volume growth, and the weighted volume are equivalent for a self-shrinker by the results by Ding and Xin [11, and the authors [6]. For self-expanders, the situation is different. For instance, $\mathbb{R}^{n}$ has the infinite weighted volume. We estimate the lower-bound of the bottom $\lambda_{1}$ of the spectrum and use the weighted $L^{2}$-integrability of the bottom $\lambda_{1}$ of the spectrum of $\mathscr{L}$ (Theorem 1.1) to discuss the rigidity of the lower bound. More precisely, we prove that

Theorem 1.2. For a complete $n$-dimensional properly immersed self-expander $\Sigma$ in $\mathbb{R}^{n+k}, k \geq 1$, the bottom of the spectrum of the drifted Laplacian 
$\mathscr{L}=\Delta+\frac{1}{2}\langle x, \nabla \cdot\rangle$, i.e. the first weighted $L^{2}$ eigenvalue of $\mathscr{L}$, satisfies

$$
\lambda_{1} \geq \frac{n}{2}
$$

The equality in (1.3) holds if and only if $\Sigma$ is the Euclidian subspace $\mathbb{R}^{n}$ through the origin.

Further in the case of self-expander hypersurfaces, using Theorem 1.1 and a Simons' type equation for the mean curvature $H$, we may obtain that

Theorem 1.3. If $\Sigma$ is a complete properly immersed self-expander hypersurface in $\mathbb{R}^{n+1}$, then the bottom $\lambda_{1}$ of the spectrum of the drifted Laplacian $\mathscr{L}=\Delta-\langle\nabla f, \nabla \cdot\rangle$ on $\Sigma$, i.e. the first weighted $L^{2}$ eigenvalue of $\mathscr{L}$ satisfies

$$
\lambda_{1} \geq \frac{n}{2}+\inf _{x \in \Sigma} H^{2}
$$

where $H$ is the mean curvature of $\Sigma$. The equality in (1.4) holds if and only if $\Sigma$ is the hyperplane $\mathbb{R}^{n}$ through the origin.

Observe that Theorem 1.2 can be implied by Theorem 1.3 when $\Sigma$ is a hypersurface.

A hypersurface is called mean convex if its mean curvature is non-negative. In [6], Colding and Minicozzi proved a complete embedded mean-convex self-shrinker hypersurface in $\mathbb{R}^{n+1}$ with polynomial volume growth must be cylinders $\mathbb{S}^{m} \times \mathbb{R}^{n-m}, 0 \leq m \leq n$. For translating solutions of MCF, i.e. translators $H=-\langle w, \mathbf{n}\rangle$, where $w$ is the constant vector in $\mathbb{R}^{n+1}$, in 21], Tasayco and the second author in the present paper proved that an immersed nonflat translator $\Sigma^{n}$ in $R^{n+1}, n=2,3$ is a grim hyperplane if and only if it is mean convex and there exists a constant $C>0$ such that $\int_{B_{R}}|A|^{2} e^{\langle x, w\rangle} d \sigma \leq C R^{2}$, for all $R$ sufficiently large, where $B_{R}$ denotes the geodesic ball of $\Sigma$. Their result is sharp in the sense that grim hyperplanes for $n \geq 4$ do not satisfy the growth condition on the integral of $|A|$ given in the above. For self-expanders, it is known that there is no any compact one and there are nonflat examples of mean-convex self-expanders (see, for instance, [1], [12]). Very recently, Fong and McGrath [13. showed that mean-convex self-expanders which are asymptotic to $O(n)$-invariant cones are rotationally symmetric. On mean convex self-expanders, by using Lemma 1 in [21] and Simons' type equations, we prove the following result:

Theorem 1.4. Let $\Sigma$ be a complete $n$-dimensional, $n \geq 2$, properly immersed mean convex self-expander in $\mathbb{R}^{n+1}$ and let $B_{R}$ denote the geodesic ball of $\Sigma$ of radius $R$ centered in a fixed point in $\Sigma$. Suppose that $h(t)$ is a positive function on $[\delta, \infty)$, for some $\delta>0$, satisfying $\frac{t}{h(t)}$ is nonincreasing on $[\delta, \infty)$ and

$$
\int_{\delta}^{\infty} \frac{t}{h(t)} d t<\infty
$$


If there exists a constant $C>0$ such that the square $|A|^{2}$ of the norm of the second fundamental form of $\Sigma$ satisfies

$$
\int_{B_{R}}|A|^{2} e^{\frac{|x|^{2}}{4}} d \sigma<C h(R)
$$

for all $R$ sufficiently large, then $\Sigma$ must be a hyperplane $\mathbb{R}^{n}$ through the origin.

Note that some examples of $\kappa(t)$ in Theorem 1.4 are $\kappa(t)=t^{2}, t^{2} \log t$, $t^{2}(\log t)(\log \log t)$, and so on. For instance, take $h(t)=t^{2}$, Theorem 1.4 implies the following

Corollary 1.1. Let $\Sigma$ be a complete $n$-dimensional, $n \geq 2$, properly immersed mean convex self-expanderin $\mathbb{R}^{n+1}$ and let $B_{R}$ denote the geodesic ball of $\Sigma$ of radius $R$ centered in a fixed point in $\Sigma$. If there exists a constant $C>0$ such that the square $|A|^{2}$ of the norm of the second fundamental form of $\Sigma$ satisfies

$$
\int_{B_{R}}|A|^{2} e^{\frac{|x|^{2}}{4}} d \sigma<C R^{2},
$$

for all $R$ sufficiently large, then $\Sigma$ must be a hyperplane $\mathbb{R}^{n}$ through the origin.

With the weight $e^{\frac{|x|^{2}}{4}}$, inequality (1.7) is a kind of asymptotic flatness condition.

In the final part of this paper, we study the $L$-stability operator for selfexpanders, which is the Schördinger operator

$$
L=\mathscr{L}+|A|^{2}-\frac{1}{2}=\Delta+\frac{1}{2}\langle x, \nabla \cdot\rangle+|A|^{2}-\frac{1}{2} .
$$

$L$-stability (see Section 21) means that the second variation of its weighted volume is nonnegative for any compactly supported normal variation (see, e.g., 3, [10]). Recall that there is no weighted-stable self-shrinkers with polynomial volume growth $(9])$. Unlike self-shrinkers, the $L$-stability is not a rigid property for self-expanders. The self-expander graphs are weightedvolume minimizing and hence $L$-stable. Also the mean convex self-expanders are $L$-stable (see Theorem 1.5). In spite of this, we may still study the bottom $\mu_{1}$ of the spectrum of the operator $L$. Since a self-expander is noncompact, $\mu_{1}$ may not be the lowest weighted $L^{2}$-eigenvalue for $L$ and also $\mu_{1}$ may take $-\infty$. We give an upper bound inequality for $\mu_{1}$ compared to the result of Colding-Minicozzi for self-shrinkers (Theorem 9.2, 8]) . More precisely, we obtain the following result:

Theorem 1.5. Let $\Sigma$ be a complete $n$-dimensional properly immersed selfexpander hypersurface. Then the bottom $\mu_{1}$ of the spectrum of the L-stability operator $L$ and the bottom $\lambda_{1}$ of the spectrum of the drifted Laplacian $\mathscr{L}$ 
satisfy

$$
\mu_{1} \leq \lambda_{1}+\frac{1}{2}
$$

The equality holds if and only if $\Sigma$ is a hyperplane through the origin.

Further, if $\Sigma$ is mean convex, then

$$
1 \leq \mu_{1} \leq \lambda_{1}+\frac{1}{2}
$$

The rest of this paper is organized as follows: In Section 2 some definitions and notations are given, in Section 3 we prove the results on the spectrum and bottom of the spectrum of the drifted Laplacian $\mathscr{L}$, in Section 4 we prove the rigidity theorem of the mean convex self-expanders and finally in Section 5 we prove the inequality on the bottom of spectrum of $L$-stability operator $L$ and the bottom of spectrum of the drifted Laplacian $\mathscr{L}$.

\section{Definitions AND NOTATION}

For the convenience on some computations in this paper and simplicity of notations, we give the notation of $f$-minimal submanifolds (see, e.g. (4)).

Assume that $(M, \bar{g})$ is a smooth $(n+k)$-dimensional Riemannian manifold and $f$ is a smooth function on $M$. A smooth metric measure space is a triple $\left(M, \bar{g}, e^{-f} d v\right)$ with a weighted volume form $e^{-f} d v$ on $M$. Here $d v$ denote the volume element of $M$ induced by the metric $\bar{g}$.

Let $i: \Sigma^{n} \rightarrow\left(M^{n+k}, \bar{g}\right)$ denote the smooth immersion of an $n$-dimensional submanifold $\Sigma$ into $M$. Then $\left(M^{n+k}, \bar{g}\right)$ induces a metric, denoted by $g$ on $\Sigma$, such that $i$ is an isometric immersion. Let $d \sigma$ denote the volume element of $(\Sigma, g)$. Then the function $f$ restricted on $\Sigma$, still denoted by $f$, induces a weighted volume element $e^{-f} d \sigma$ on $\Sigma$ and thus a smooth metric measure space $\left(\Sigma, g, e^{-f} d \sigma\right)$.

The isometric immersion $i:\left(\Sigma^{n}, g\right) \rightarrow\left(M^{n+k}, \bar{g}\right)$ is said to be properly immersed if, for any compact subset $\Omega$ in $M$, the pre-image $i^{-1}(\Omega)$ is compact in $\Sigma$.

In this paper, unless otherwise specified, the notations with a bar denote the quantities corresponding the metric $\bar{g}$ on $M$. For instance $\bar{\nabla}$ and $\bar{\nabla}^{2}$ denote the Levi-Civita connection, and Hession of $(M, \bar{g})$ respectively. On the other hand, the notations like $\nabla$ denote the quantities corresponding the intrinsic metric $g$ on $\Sigma$. For instance $\nabla, \Delta$ denote the Levi-Civita connection and the Laplacian on $(\Sigma, g)$ respectively.

Let $A$ denote the second fundamental form of $\Sigma$. The mean curvature vector $\mathbf{H}(p)$ of $\Sigma$ at $p \in \Sigma$ is defined by

$$
\mathbf{H}(p):=\sum_{i=1}^{n}\left(\bar{\nabla}_{e_{i}} e_{i}\right)^{\perp}=\sum_{i=1}^{n} A\left(e_{i}, e_{i}\right),
$$

where $\left\{e_{1}, e_{2}, \cdots, e_{n}\right\}$ is a local orthonormal frame of $\Sigma$ at $p$ and $\perp$ denotes the projection onto the normal bundle of $\Sigma$. 
The weighted volume of a measurable subset $S \subset \Sigma$ is defined by

$$
V_{f}(S):=\int_{S} e^{-f} d \sigma
$$

Definition 2.1. The weighted mean curvature vector $\mathbf{H}_{f}$ of the submanifold $(\Sigma, g)$ is defined by

$$
\mathbf{H}_{f}:=\mathbf{H}+(\bar{\nabla} f)^{\perp} .
$$

A submanifold $(\Sigma, g)$ is called $f$-minimal if its weighted mean curvature $\mathbf{H}_{f}$ vanishes identically, or equivalently if it satisfies

$$
\mathbf{H}=-(\bar{\nabla} f)^{\perp} .
$$

In the case of hypersurfaces, the mean curvature of $\Sigma$ is defined by

$$
\mathbf{H}=-H \mathbf{n},
$$

where $\mathbf{n}$ is the unit normal field on $\Sigma$.

From Definition 2.1, the hypersurface $\Sigma$ is $f$-minimal if and only if

$$
H_{f}=H-\langle\bar{\nabla} f, \mathbf{n}\rangle=0 \text {, }
$$

or equivalently

$$
H=\langle\bar{\nabla} f, \mathbf{n}\rangle .
$$

It is known that an $f$-minimal submanifold is a critical point of the weighted volume functional defined in (2.1). On the other hand, it is also a minimal submanifold under the conformal metric $\tilde{g}=e^{-\frac{2}{n} f} \bar{g}$ on $M$ (see, e.g. [3], [4]).

When $(M, \bar{g})$ is the Euclidean space $\left(\mathbb{R}^{n+k}, g_{0}\right)$, we have some interesting examples of $f$-minimal submanifolds:

Example 2.1. If $f=\frac{|x|^{2}}{4},-\frac{|x|^{2}}{4}$, and $-\langle x, w\rangle$ respectively, where $w \in \mathbb{R}^{n+k}$ is a constant vector, an $n$-dimensional $f$-minimal submanifold $\Sigma$ is a selfshrinker, self-expander and translator for MCF in the Euclidian space $\mathbb{R}^{n+k}$ respectively.

For $\left(\Sigma, g, e^{-f} d \sigma\right)$, let $L^{2}\left(\Sigma, e^{-f} d \sigma\right)$ denote the space of square-integrable functions on $\Sigma$ (not necessarily a submanifold) with respect to the measure $e^{-f} d \sigma$. The weighted $L^{2}$ inner product of functions $u$ and $v$ in $L^{2}\left(\Sigma, e^{-f} d \sigma\right)$ is defined by

$$
\langle u, v\rangle_{L^{2}\left(\Sigma, e^{-f} d \sigma\right)}=\int_{\Sigma} u v e^{-f} d \sigma .
$$

The drifted Laplacian on $\Sigma$ is defined by

$$
\Delta_{f}=\Delta-\langle\nabla f, \nabla \cdot\rangle .
$$

It is well known that $\Delta_{f}$ is a densely defined self-adjoint operator in $L^{2}\left(\Sigma, e^{-f} d \sigma\right)$, i.e. for $u$ and $v$ in $C_{0}^{\infty}(\Sigma)$, it holds that

$$
\int_{\Sigma}\left(\Delta_{f} u\right) v e^{-f} d \sigma=-\int_{\Sigma}\langle\nabla u, \nabla v\rangle e^{-f} d \sigma
$$


We recall some facts in spectral theory (see more details in, e.g. [15], [19]). Consider the Schrödinger operator on $\Sigma$ :

$$
S=\Delta_{f}+q, \quad q \in L_{l o c}^{\infty}(\Sigma) .
$$

The weighted $L^{2}$ spectrum of $S$ is called the spectrum of $S$ for short whenever there is no confusion. The bottom $s_{1}$ of the spectrum of $S$ can be characterized by

$$
s_{1}=\inf \left\{\frac{\int_{\Sigma}\left(|\nabla \varphi|^{2}-q \varphi^{2}\right) e^{-f} d \sigma}{\int_{\Sigma} \varphi^{2} e^{-f} d \sigma} ; \varphi \in C_{0}^{\infty}(\Sigma), \int_{\Sigma} \varphi^{2} e^{-f} d \sigma \neq 0\right\}
$$

A number $s$ is said to be a weighted $L^{2}$ eigenvalue to $S$ if there exists a smooth nonzero function $u \in L^{2}\left(\Sigma, e^{-f} d \sigma\right)$ satisfying

$$
S u+s u=0,
$$

The function $u$ in (2.8) is called the weighted $L^{2}$ eigenfunction associated to $s$.

In general, if $\Sigma$ is non-compact, the bottom $s_{1}$ may not be a weighted $L^{2}$ eigenvalue and may be $-\infty$. The spectral theory says that if the spectrum of $S$ is discrete, the spectrum is the set of all the weighted $L^{2}$ eigenvalues of $S$, counted with multiplicity, which is an increasing sequence

$$
s_{1}<s_{2} \leq s_{3} \leq \cdots
$$

with $s_{i} \rightarrow \infty$ as $i \rightarrow \infty$. Further, the variational characterization of $s_{i}$ states that the bottom $s_{1}$ of spectrum of $S$ is just the first weighted $L^{2}$ eigenvalue with multiplicity 1 .

Now we give special notations for self-expanders. In the following, unless otherwise specified, let $\Sigma$ be an $n$-dimensional self-expander in $\mathbb{R}^{n+k}, k \geq 1$, that is, $\Sigma$ satisfies the equation

$$
\mathbf{H}=\frac{x^{\perp}}{2}
$$

In the case of codimension $1, \Sigma$ is a self-expander if and only if the mean curvature $H$ satisfies that

$$
H=-\frac{1}{2}\langle x, \mathbf{n}\rangle
$$

Observe that a self-expander $\Sigma$ can be viewed as an $f$-minimal submanifold in $\mathbb{R}^{n+k}$ by taking $f=-\frac{|x|^{2}}{4}$ in equation (2.3).

The weighted volume of a measurable subset $S \subset \Sigma$ is given by

$$
V_{f}(S):=\int_{S} e^{\frac{|x|^{2}}{4}} d \sigma
$$

The weighted $L^{2}$ inner product of functions $u$ and $v$ in $L^{2}\left(\Sigma, e^{\frac{|x|^{2}}{4}} d \sigma\right)$ is defined by

$$
\langle u, v\rangle_{L^{2}\left(\Sigma, e^{\frac{|x|^{2}}{4}} d \sigma\right)}=\int_{\Sigma} u v e^{\frac{|x|^{2}}{4}} d \sigma
$$


Denote by $\mathscr{L}$ the drifted Laplacian on $\Sigma$, i.e. $\mathscr{L}=\Delta+\frac{1}{2}\langle x, \nabla \cdot\rangle$.

The bottom $\lambda_{1}$ of the spectrum of $\mathscr{L}$ can be given by

$$
\lambda_{1}=\inf \left\{\frac{\int_{\Sigma}|\nabla \varphi|^{2} e^{\frac{|x|^{2}}{4}} d \sigma}{\int_{\Sigma} \varphi^{2} e^{\frac{|x|^{2}}{4}} d \sigma} ; \varphi \in C_{0}^{\infty}(\Sigma), \int_{\Sigma} \varphi^{2} e^{\frac{|x|^{2}}{4}} d \sigma \neq 0\right\} .
$$

From (2.12), $\lambda_{1}$ is nonnegative. For self-expanders, the stability operator, which appears in the second variation of the weighted volume, is a Schrödinger operator given by

$$
L=\mathscr{L}+|A|^{2}-\frac{1}{2}=\Delta+\frac{1}{2}\langle x, \nabla \cdot\rangle+|A|^{2}-\frac{1}{2} .
$$

Definition 2.2. A self-expander $\Sigma$ is said to be L-stable if the following inequality holds for all $\varphi \in C_{0}^{\infty}(\Sigma)$,

$$
-\int_{\Sigma} \varphi(L \varphi) e^{\frac{|x|^{2}}{4}} d \sigma=\int_{\Sigma}\left(|\nabla \varphi|^{2}-\left(|A|^{2}-\frac{1}{2}\right) \varphi^{2}\right) e^{\frac{|x|^{2}}{4}} d \sigma \geq 0 .
$$

$L$-stability of $\Sigma$ is equivalent to that the second variation of its weighted volume is nonnegative for any compactly supported normal variation. Denote the bottom of the spectrum of $L$ by $\mu_{1}$. $L$-stability means $\mu_{1} \geq 0$.

\section{Spectral Properties of the DRIFted Laplacian}

In this section, we show the discreteness of the spectrum of the drifted Laplacian $\mathscr{L}=\Delta+\frac{1}{2}\langle x, \nabla \cdot\rangle$ and study the bottom of the spectrum of $\mathscr{L}$ for complete properly immersed self-expanders. We start by giving the following identities:

Lemma 3.1. For a manifold $(\Sigma, g)$ (not necessarily submanifold),

$$
\Delta_{f}\left(u e^{h}\right)=e^{h}\left\{\Delta_{f-2 h} u+[\Delta h+\langle\nabla(h-f), \nabla h\rangle] u\right\},
$$

where $f$ and $h$ are smooth functions on $\Sigma$.

In particular, if $h=f$,

$$
\Delta_{f}\left(u e^{f}\right)=e^{f}\left[\Delta_{(-f)} u+(\Delta f) u\right] .
$$

Proof. The results come from the direct computations.

$$
\begin{aligned}
& \nabla\left(u e^{h}\right)=e^{h}(\nabla u+u \nabla h) \\
& \Delta\left(u e^{h}\right)=e^{h}\left[\Delta u+2\langle\nabla u, \nabla h\rangle+u\left(\Delta h+|\nabla h|^{2}\right)\right]
\end{aligned}
$$

By (3.3) and (3.4), we have

$$
\begin{aligned}
\Delta_{f}\left(u e^{h}\right)= & e^{h}\left[\Delta u+2\langle\nabla u, \nabla h\rangle+u\left(\Delta h+|\nabla h|^{2}\right)\right] \\
& \quad-e^{h}(\langle\nabla f, \nabla u\rangle+u\langle\nabla f, \nabla h\rangle) \\
= & e^{h}[\Delta u-\langle\nabla(f-2 h), \nabla u\rangle+u(\Delta h+\langle\nabla h, \nabla(h-f)\rangle]
\end{aligned}
$$


Recall that a function $h: \Sigma \rightarrow \mathbb{R}$ is said to be proper if, for any bounded closed subset $I \subset \mathbb{R}$, the inverse image $h^{-1}(I)$ is compact in $\Sigma$. Now we prove Theorem 1.1 .

Proof of Theorem 1.1. Take $f=-\frac{|x|^{2}}{4}$. Consider the unitary isomorphism

$$
U: L^{2}(\Sigma, d \sigma) \rightarrow L^{2}\left(\Sigma, e^{-f} d \sigma\right)=L^{2}\left(\Sigma, e^{\frac{|x|^{2}}{4}} d \sigma\right)
$$

given by $U u=u e^{\frac{f}{2}}$. Take $h=\frac{f}{2}$ in (3.1) and note $\mathscr{L}=\Delta_{f}$. We have

$$
\mathscr{L}=U T U^{-1}, \quad T=\Delta+\frac{1}{2} \Delta f-\frac{1}{4}|\nabla f|^{2} .
$$

By the spectral theory, the discreteness of the spectrum of $\mathscr{L}$ and the discreteness of the spectrum of $T$ are equivalent.

Note that $\mathbf{H}=-(\bar{\nabla} f)^{\perp}$ and $f=-\frac{|x|^{2}}{4}$. It holds that

$$
\Delta f=\sum_{i=1}^{n} \bar{\nabla}^{2} f\left(e_{i}, e_{i}\right)+\langle\bar{\nabla} f, \mathbf{H}\rangle=-\frac{n}{2}-|\mathbf{H}|^{2} .
$$

Since Sigma is a self-expander, we have

$$
\begin{aligned}
\frac{1}{4}|\nabla f|^{2}-\frac{1}{2} \Delta f & =\frac{1}{4}|\bar{\nabla} f|^{2}+\frac{1}{4}\left|(\bar{\nabla} f)^{\perp}\right|^{2}+\frac{n}{4} \\
& =\frac{1}{4}|x|^{2}+\frac{1}{16}\left|x^{\perp}\right|^{2}+\frac{n}{4} \\
& \geq \frac{1}{4}|x|^{2}+\frac{n}{4}
\end{aligned}
$$

Since $\Sigma$ is properly immersed in $\mathbb{R}^{n+k}$, the function $\frac{1}{4}|x|^{2}+\frac{n}{4}$ restricted on $\Sigma$ is proper and tends to $\infty$ when the intrinsic distance $d^{\Sigma}(p, x)$ of $\Sigma$ tends $\infty$, where $p \in \Sigma$ fixed. (3.7) implies that $\frac{1}{4}|\nabla f|^{2}-\frac{1}{2} \Delta f$ also tends to $\infty$ when $d^{\Sigma}(p, x) \rightarrow \infty$. This implies that the spectrum of the operator $T$ is discrete (e.g. [19] page 120). Hence, the spectrum of $\mathscr{L}$ is discrete.

By the discreteness of the spectrum of the drifted Laplacian $\mathscr{L}$, the variational character of the first weighted $L^{2}$ eigenvalue of $\mathscr{L}$ implies that it is just the bottom $\lambda_{1}$ of the spectrum of $\mathscr{L}$.

We consider the spectrum of $\mathscr{L}$ on $\mathbb{R}^{n}$ and obtain that

Proposition 3.1. i) The spectrum of $\mathscr{L}=\Delta+\frac{1}{2}\langle x, \nabla \cdot\rangle$ on $\mathbb{R}^{n}$ is discrete and the weighted $L^{2}$ eigenvalues of $\mathscr{L}$, counted by multiplicity, are the following

$$
\lambda=\frac{n}{2}+\frac{1}{2} \sum_{i=1}^{n} k_{i}, \quad k_{i} \in\{0\} \cup \mathbb{N},
$$


where $\frac{k_{i}}{2}$, are the weighted $L^{2}$ eigenvalues of the operator $\frac{d^{2}}{d x_{i}^{2}}-\frac{x_{i}}{2} \frac{d}{d x_{i}}, x_{i} \in$ $\mathbb{R}$ with respect to the weighted measure $e^{-\frac{x_{i}^{2}}{4}} d x_{i}$, that is, the weighted $L^{2}$ eigenfunctions associated to $\frac{k_{i}}{2}$ are the Hermite polynomials $H_{k_{i}}\left(\frac{x_{i}}{2}\right)$.

ii) The weighted $L^{2}$ eigenfunction associated to $\lambda$, counted by multiplicity, is $\Phi=\prod_{i=1}^{n} H_{k_{i}}\left(\frac{x_{i}}{2}\right) e^{-\frac{|x|^{2}}{4}}$, where $\Pi_{i=1}^{n} H_{k_{i}}\left(\frac{x_{i}}{2}\right)$ are the products of the corresponding Hermite polynomials.

iii) The bottom $\lambda_{1}$ of the spectrum of $\mathscr{L}$, that is, the first weighted $L^{2}$ eigenvalue of $\mathscr{L}$ is $\frac{n}{2}$ with multiplicity 1 ;

Proof. Take $f=-\frac{|x|^{2}}{4}$ in (3.2). Note, $\mathscr{L}=\Delta+\frac{1}{2}\langle x, \nabla \cdot\rangle$ and $\mathcal{L}=\Delta-$ $\frac{1}{2}\langle x, \nabla \cdot\rangle$.

$$
\mathscr{L}\left(u e^{-\frac{|x|^{2}}{4}}\right)=e^{-\frac{|x|^{2}}{4}}\left(\mathcal{L}-\frac{n}{2}\right) u
$$

Observe that $u \in L^{2}\left(\mathbb{R}^{n}, e^{-\frac{|x|^{2}}{4}} d \sigma\right)$ if and only if $u e^{-\frac{|x|^{2}}{4}} \in L^{2}\left(\mathbb{R}^{n}, e^{\frac{|x|^{2}}{4}} d \sigma\right)$.

$$
\mathcal{L}=\sum_{i=1}^{n}\left(\frac{\partial^{2}}{\partial x_{i}^{2}}-\frac{x_{i}}{2} \frac{\partial}{\partial x_{i}}\right)
$$

For the operator $\frac{d^{2}}{d t^{2}}-\frac{t}{2} \frac{d}{d t}, t \in \mathbb{R}$, it is known that its spectrum on $L^{2}\left(\mathbb{R}, e^{-\frac{t^{2}}{4}} d t\right)$ is discrete and the Hermite polynomials $H_{k}\left(\frac{x}{2}\right)$ are orthonormal eigenfunctions associated to $\frac{k}{2}, k \in\{0\} \cup \mathbb{N}$, which form a complete orthonormal system for space $L^{2}\left(\mathbb{R}, e^{-\frac{t^{2}}{4}} d t\right)$. By this fact and (3.10), one can verify that the products $\Pi_{i=1}^{n} H_{k_{i}}\left(\frac{x_{i}}{2}\right)$ of $n$ eigenfunctions $H_{k_{i}}\left(\frac{x_{i}}{2}\right)$ of $\frac{d^{2}}{d x_{i}^{2}}-\frac{x_{i}}{2} \frac{d}{d x_{i}}$ respectively, $1 \leq i \leq n, k_{i} \in\{0\} \cup \mathbb{N}$, are the eigenfunctions of $\mathcal{L}$ associated to the eigenvalue $\frac{1}{2} \sum_{i=1}^{n} k_{i}$ and, by a standard argument in functional analysis,, form a complete system for the space $L^{2}\left(\mathbb{R}^{n}, e^{-\frac{|x|^{2}}{4}} d \sigma\right)$.

By (3.9), $\mathscr{L}$ has the discrete spectrum and the weighted $L^{2}$ eigenvalues, counted by multiplicity, are the following

$$
\lambda=\frac{n}{2}+\frac{1}{2} \sum_{i=1}^{n} k_{i}, \quad k_{i} \in\{0\} \cup \mathbb{N},
$$

and associated eigenfunctions are $\Phi=\prod_{i=1}^{n} H_{k_{i}}\left(\frac{x_{i}}{2}\right) e^{-\frac{|x|^{2}}{4}}$, which form a complete orthonormal system for the space $L^{2}\left(\mathbb{R}^{n}, e^{\frac{|x|^{2}}{4}} d \sigma\right)$.

Now we prove Theorem 1.2 in which we study the universal lower bound for the bottom of the spectrum of $\mathscr{L}$ for self-expanders. 
Proof of Theorem 1.2. Taking $u \equiv 1$ and $f=-\frac{|x|^{2}}{4}$ in (3.2) yields

$$
\mathscr{L}\left(e^{-\frac{|x|^{2}}{4}}\right)=e^{-\frac{|x|^{2}}{4}}\left[\left(\Delta\left(-\frac{|x|^{2}}{4}\right)\right] .\right.
$$

Using (3.6), we have the positive function $v=e^{-\frac{|x|^{2}}{4}}>0$ satisfying that

$$
\mathscr{L} v+\left(\frac{n}{2}+|\mathbf{H}|^{2}\right) v=0 .
$$

It is well known that (3.12) implies that for any $\varphi \in C_{0}^{\infty}(\Sigma)$,

$$
\int_{\Sigma}\left[|\nabla \varphi|^{2}-\left(\frac{n}{2}+|\mathbf{H}|^{2}\right) \varphi^{2}\right] e^{\frac{|x|^{2}}{4}} d \sigma \geq 0
$$

Then the bottom $\lambda_{1}$ of spectrum of $\mathscr{L}$ satisfies that

$$
\lambda_{1}=\inf _{\varphi \in C_{0}^{\infty}(\Sigma), \int \varphi^{2} e^{\frac{|x|^{2}}{4}} d \sigma \neq 0} \frac{\int|\nabla \varphi|^{2} e^{\frac{|x|^{2}}{4}} d \sigma}{\int \varphi^{2} e^{\frac{|x|^{2}}{4}} d \sigma} \geq \frac{n}{2}+\left.\inf _{x \in \Sigma}|\mathbf{H}|\right|^{2} \geq \frac{n}{2} .
$$

So we have proved that the inequality holds.

Now we assume that the equality $\lambda_{1}=\frac{n}{2}$ holds. By Theorem 1.1, the spectrum of $\mathscr{L}$ of $\Sigma$ is discrete and $\lambda_{1}$ is the first weighted $L^{2}$ eigenvalue of $\mathscr{L}$. Then there exists the first eigenfunction $u>0$ such that $u \in W^{1,2}(\Sigma)$ and

$$
\mathscr{L} u+\lambda_{1} u=0
$$

Note $v=e^{-\frac{|x|^{2}}{4}}>0$. By (3.12),

$$
\mathscr{L} \log v=\frac{\mathscr{L} v}{v}-\frac{|\nabla v|^{2}}{v^{2}}=-\frac{n}{2}-|\mathbf{H}|^{2}-|\nabla \log v|^{2}
$$

Take $\phi \in C_{0}^{\infty}(\Sigma)$.

$$
\begin{aligned}
\int_{\Sigma}\left(\frac{n}{2}+|\mathbf{H}|^{2}+|\nabla \log v|^{2}\right) \phi^{2} e^{\frac{|x|^{2}}{4}} d \sigma & =-\int_{\Sigma} \phi^{2}(\mathscr{L} \log v) e^{\frac{|x|^{2}}{4}} d \sigma \\
& =\int_{\Sigma}\left\langle\nabla \phi^{2}, \nabla \log v\right\rangle e^{\frac{|x|^{2}}{4}} d \sigma
\end{aligned}
$$

Since

$$
\int_{\Sigma}\left\langle\nabla \phi^{2}, \nabla \log w\right\rangle e^{\frac{|x|^{2}}{4}} d \sigma \leq \int_{\Sigma}|\nabla \phi|^{2} e^{\frac{|x|^{2}}{4}} d \sigma+\int_{\Sigma}|\nabla \log w|^{2} \phi^{2} e^{\frac{|x|^{2}}{4}} d \sigma
$$

(3.17) implies that

$$
\int_{\Sigma}\left(\frac{n}{2}+|\mathbf{H}|^{2}\right) \phi^{2} e^{\frac{|x|^{2}}{4}} d \sigma \leq \int_{\Sigma}|\nabla \phi|^{2} e^{\frac{|x|^{2}}{4}} d \sigma
$$

Choose $\phi=\varphi_{j} u$, where $\varphi_{j}$ are the non-negative cut-off functions satisfying that $\varphi_{j}$ is 1 on $B_{j},|\nabla \varphi| \leq 1$ on $B_{j+1} \backslash B_{j}$, and $\varphi=0$ on $\Sigma \backslash B_{j+1}$. 
Substitute $\phi$ in (3.18):

$$
\left.\int_{\Sigma}\left|\left(\frac{n}{2}+|\mathbf{H}|^{2}\right) \varphi_{i}^{2} u^{2} e^{\frac{|x|^{2}}{4}} d \sigma \leq \int_{\Sigma}\right| \nabla\left(\varphi_{j} u\right)\right|^{2} e^{\frac{|x|^{2}}{4}} d \sigma .
$$

Note that

$$
\int_{\Sigma}\left|\nabla\left(\varphi_{j} u\right)\right|^{2} e^{\frac{|x|^{2}}{4}} d \sigma \leq 2 \int_{\Sigma} \varphi_{j}^{2}|\nabla u|^{2} e^{\frac{|x|^{2}}{4}} d \sigma+2 \int_{\Sigma}\left|\nabla \varphi_{j}\right|^{2} u^{2} e^{\frac{|x|^{2}}{4}} d \sigma
$$

and $u \in W^{1,2}(\Sigma)$. Letting $j \rightarrow \infty$ in (3.20) and using the monotone convergence theorem,

$$
\int_{\Sigma}\left|\nabla\left(\varphi_{j} u\right)\right|^{2} e^{\frac{|x|^{2}}{4}} d \sigma \rightarrow \int_{\Sigma}|\nabla u|^{2} e^{\frac{|x|^{2}}{4}} d \sigma .
$$

Letting $j \rightarrow \infty$ in (3.19) and using the monotone convergence theorem again, we have

$$
\begin{aligned}
\int_{\Sigma}\left(\frac{n}{2}+|\mathbf{H}|^{2}\right) u^{2} e^{\frac{|x|^{2}}{4}} d \sigma & \leq \int_{\Sigma}|\nabla u|^{2} e^{\frac{|x|^{2}}{4}} d \sigma \\
& =\frac{n}{2} \int_{\Sigma} u^{2} e^{\frac{|x|^{2}}{4}} d \sigma .
\end{aligned}
$$

Hence $|\mathbf{H}|=\mathbf{0}$. Since $\mathbf{H}=\frac{1}{2} x^{\perp}=0 . \Sigma$ is an $n$-dimensional minimal cone. Since $\Sigma$ is smooth, $\Sigma$ must be $\mathbb{R}^{n}$. By the self-expander equation, it passes through the origin.

It is known that a submanifold in $\mathbb{R}^{n+k}, k>0$, is minimal if and only if the coordinate functions restricted on $\Sigma$ are the harmonic functions. For self-expanders, there are similar properties:

Proposition 3.2. An $n$-dimensional immersed submanifold $\Sigma$ in $\mathbb{R}^{n+k}, k>$ 0 , is a self-expander if and only if the coordinate functions restricted on $\Sigma$ are eigenfunctions of the drifted Laplacian $\mathscr{L}$. Moreover, they are corresponding the eigenvalue $-\frac{1}{2}$, i.e. they satisfy

$$
\mathscr{L} x_{i}-\frac{1}{2} x_{i}=0
$$

for all $i=1,2, \ldots, n+k$.

Proof. Take $f=-\frac{|x|^{2}}{4}$.

$$
\begin{aligned}
\mathscr{L} x_{i} & =\Delta x_{i}-\left\langle\nabla f, \nabla x_{i}\right\rangle \\
& =\sum_{j=1}^{n} \bar{\nabla}^{2} x_{i}\left(e_{j}, e_{j}\right)+\left\langle\mathbf{H}, \bar{\nabla} x_{i}\right\rangle-\left\langle\nabla f, \nabla x_{i}\right\rangle \\
& =\left\langle\mathbf{H}+(\bar{\nabla} f)^{\perp}, \bar{\nabla} x_{i}\right\rangle-\left\langle\bar{\nabla} f, \bar{\nabla} x_{i}\right\rangle \\
& =\left\langle\mathbf{H}_{f}, \bar{\nabla} x_{i}\right\rangle+\frac{x_{i}}{2} .
\end{aligned}
$$


In the above $\left\{e_{1}, e_{2}, \cdots, e_{n}\right\}$ is an orthonormal basis of the tangent space $T_{p} \Sigma, p \in \Sigma$.

$$
\mathscr{L} x_{i}-\frac{x_{i}}{2}=\left\langle\mathbf{H}_{f}, \bar{\nabla} x_{i}\right\rangle .
$$

So the left-hand side is zero for all $i$ if and only only if $\left\langle\mathbf{H}_{f}, e_{i}\right\rangle=0$ for $i$ which is equivalent to $\mathbf{H}_{f}=0$.

Remark 3.1. The above results hold locally. For properly immersed submanifolds, it has been known that the coordinate functions are the weighted $L^{2}$ eigenfunctions for the corresponding drifted Laplacian for a self-shrinker. For self-expanders, the corresponding drifted Laplacian still has discrete spectrum but the coordinate functions are not the weighted $L^{2}$ eigenfunctions.

In the rest of this section, we discuss self-expanders of codimension 1 whose equation is

$$
H=-\frac{1}{2}\langle x, \mathbf{n}\rangle \text {. }
$$

Recall that in [2], Mejia and the authors calculated the Simons' type equations for general $f$-minimal hypersurfaces as follows.

Proposition 3.3. (Propostion 1 in [2]) Let $\left(\Sigma^{n}, g\right)$ be an $f$-minimal hypersurface isometrically immersed in a smooth metric measure space $\left(M, \bar{g}, e^{-f} d \mu\right)$. Then the mean curvature $H$ of $\Sigma$ satisfies that

$$
\begin{aligned}
\Delta_{f} H= & 2 \sum_{i=1}^{n}\left(\bar{\nabla}^{3} f\right)_{i \nu i}-\sum_{i=1}^{n}\left(\bar{\nabla}^{3} f\right)_{\nu i i}+2 \sum_{i, j=1}^{n} a_{i j}\left(\bar{\nabla}^{2} f\right)_{i j} \\
& -\overline{\operatorname{Ric}}_{f}(\nu, \nu) H-|A|^{2} H,
\end{aligned}
$$

where $\left\{e_{1}, \ldots, e_{n}\right\}$ is a local orthonormal frame field on $\Sigma, \nu$ denotes the unit normal to $\Sigma, a_{i j}=A\left(e_{i}, e_{j}\right)$, and $\overline{\operatorname{Ric}}_{f}=\overline{\operatorname{Ric}}+\bar{\nabla}^{2} f$.

Proposition 3.4. (Corollary 3 in [2]) Let $\left(M^{n+1}, \bar{g}, e^{-f} d \mu\right)$ be a smooth metric metric space satisfying $\overline{R i c}_{f}=C \bar{g}$, where $C$ is a constant. If $(\Sigma, g)$ is an $f$-minimal hypersurface isometrically immersed in $M$, then it holds that on $\Sigma$

$$
\begin{aligned}
\frac{1}{2} \Delta_{f}|A|^{2}= & |\nabla A|^{2}+C|A|^{2}-|A|^{4}+\sum_{i, j=1}^{n} a_{i j} \bar{R}_{i \nu j \nu ; \nu} \\
& -2 \sum_{i, j, k=1}^{n} a_{i j} a_{i k} \bar{R}_{j \nu k \nu}-2 \sum_{i, j, k, l=1}^{n} a_{i j} a_{l k} \bar{R}_{i l j k},
\end{aligned}
$$

where the notation is the same as in Propostion 3.3 .

$$
\text { Take } f=-\frac{|x|^{2}}{4} \text { and } M=\mathbb{R}^{n+k} \text { and note } H=\sum_{i=1}^{n} a_{i i},|A|^{2}=\sum_{i=1}^{n} a_{i i}^{2} \text {. It }
$$
holds that the Simons' type equations for self-expanders: 
Lemma 3.2. For self-expanders of codimension 1,

$$
\mathscr{L} H+\left(|A|^{2}+\frac{1}{2}\right) H=0 .
$$

and

$$
\frac{1}{2} \mathscr{L}|A|^{2}=|\nabla A|^{2}-\frac{1}{2}|A|^{2}-|A|^{4} .
$$

(See (3.26) also in [10]). Using (3.26), we can give

Proof of Theorem 1.3. Similar to the proof of Theorem[1.2, we have (3.14) gives

$$
\lambda_{1} \geq \frac{n}{2}+\inf _{x \in \Sigma} H^{2}
$$

Suppose that the equality $\lambda_{1}=\frac{n}{2}+\inf _{x \in \Sigma} H^{2}$ holds. Again there exists the first eigenfunction $u>0$ such that $u \in W^{1,2}(\Sigma)$ and

$$
\mathscr{L} u+\lambda_{1} u=0 .
$$

The proof of Theorem 1.2 gives

$$
\int_{\Sigma}\left(\frac{n}{2}+H^{2}\right) u^{2} e^{\frac{|x|^{2}}{4}} d \sigma \leq \int_{\Sigma}|\nabla u|^{2} e^{\frac{|x|^{2}}{4}} d \sigma=\lambda_{1} \int_{\Sigma} u^{2} e^{\frac{|x|^{2}}{4}} d \sigma .
$$

This implies that $H^{2}=\inf _{x \in \Sigma} H^{2}=C$. The Simons' type equation (3.26) says that

$$
\mathscr{L} H+\left(|A|^{2}+\frac{1}{2}\right) H=0 .
$$

It implies that $H=0$. Hence $\Sigma$ must be $\mathbb{R}^{n}$ through the origin.

\section{MeAn CONVEX SElF-EXPANDERS OF CODIMENSION 1}

A self-expander hypersurface $\Sigma$ is called mean convex if its mean curvature $H \geq 0$. Besides the hyperplane $\mathbb{R}^{n}$ through the origin, there are nontrivial examples (see, e.g. [12]). In this section, We will prove some rigidity results on mean convex self-expander hypersurfaces.

First we need the following result proved byTasayco and the second author in [21]:

Lemma 4.1. (Lemma 1 in [21]) On a complete weighted manifold $\left(M,\langle\rangle,, e^{-f} d \mathrm{voL}\right)$, assume that the functions $u, v \in C^{2}(M)$, with $u>0$ and $v \geq 0$ on $M$, satisfy

$$
\Delta_{f} u+q(x) u \leq 0 \quad \text { and } \quad \Delta_{f} v+q(x) v \geq 0
$$


where $q(x) \in C^{0}(M)$. Suppose that there exists a positive function $\kappa>0$ on $[\delta, \infty)$ for some $\delta>0$, satisfying $\frac{t}{\kappa(t)}$ is nonincreasing on $[\delta, \infty)$ and

$$
\int_{\delta}^{+\infty} \frac{t}{\kappa(t)} d t=+\infty
$$

such that

$$
\int_{B_{R}} v^{2} e^{-f} \leq \kappa(R)
$$

for all $R$ sufficiently large, where $B_{R}$ denotes the geodesic ball of radius $R$ of $\Sigma$. Then there exists a constant $C$ such that $v=C u$.

Using Lemma 4.1, we can show Theorem 1.4.

Proof of Theorem 1.4. Since $H \geq 0$ and $\mathscr{L} H+\left(|A|^{2}+\frac{1}{2}\right) H=0$, by the argument using the Harnack inequality we have $H \equiv 0$ or $H>0$ on $\Sigma$. If $H \equiv 0$, it is hyperplane $\mathbb{R}^{n}$ through the origin. Assume that $H>0$ on $\Sigma$. It holds that

$$
\mathscr{L} H+\left(|A|^{2}+\frac{1}{2}\right) H=0 .
$$

We also have the Simons' type equation (3.27) for $|A|$ :

$$
\frac{1}{2} \mathscr{L}|A|^{2}=|\nabla A|^{2}-\frac{1}{2}|A|^{2}-|A|^{4} .
$$

This and $n|A|^{2} \geq H^{2}>0$ imply that

$$
\mathscr{L}|A|=-\frac{1}{2}|A|-|A|^{3}+\frac{|\nabla A|^{2}-|\nabla| A||^{2}}{|A|} .
$$

Since $|\nabla A|^{2}-\left.|\nabla| A\right|^{2} \geq 0$, (see, e.g. Lemma $10.2[$ ] )

$$
\mathscr{L}|A|+\left(|A|^{2}+\frac{1}{2}\right)|A|=\frac{|\nabla A|^{2}-\left.|\nabla| A\right|^{2}}{|A|} \geq 0 .
$$

By (4.4), (4.7), and the hypothesis of the theorem, using Lemma 4.1, it holds that $H=C|A|$ on $\Sigma$ and

$$
\mathscr{L}|A|+\left(|A|^{2}+\frac{1}{2}\right)|A|=0 .
$$

Hence $|\nabla A|=|\nabla| A||$.

The rest of the proof is similar to the one of Huisken ([16]. See, e.g. the proof of Theorem 0.17 in [8]). We only focus on the different points of the argument. Following the proof in $[8,|\nabla A|=|\nabla| A||$ implies two possible cases: (I) If the rank of $A$ is greater than 2 , it implies that $\nabla A \equiv 0$ on $\Sigma$. Thus $|A|$ is constant on $\Sigma$. $H=C|A|>0$ says $|A|$ is a positive constant which induces a contradiction with (4.8). (II) If the rank of $A$ is 1 , then $\Sigma$ is the product of a curve $\gamma(t) \subset \mathbb{R}^{2}$ and an $(n-1)$-dimensional hyperplane. If $\gamma(t)$ is a line, it contradicts with $H>0$. If $\gamma(t)$ is not a line, it will 
contradicts the condition (1.6), since the part of the weight $e^{\frac{|x|^{2}}{4}}$ restricted on $\mathbb{R}^{n-1}$ has the growth order bigger than the one of any polynomial.

Theorem 1.4 implies Corollary 1.1 and the following

Corollary 4.1. If a complete $n$-dimensional, $n \geq 2$, immersed self-expander hypersurface $\Sigma$ is mean convex, and $\int_{\Sigma}|A|^{2} e^{\frac{|x|^{2}}{4}} d \sigma<\infty$, then $\Sigma$ must be a hyperplane $\mathbb{R}^{n}$ through the origin.

\section{The BotTom of SPECTRUM OF STABILITY OPERATOR OF SELF-EXPANDERS}

In this section, we study the bottom $\mu_{1}$ of the weighted stability operator $L=\mathscr{L}+|A|^{2}-\frac{1}{2}=\Delta+\frac{1}{2}\langle x, \nabla \cdot\rangle+|A|^{2}-\frac{1}{2}$ of a self-expander hypersurface.

Proof of Theorem 1.5. Note $\mu_{1}$ may take $-\infty$ and in this case (1.9) holds. Assume that $\mu_{1}>-\infty$. Then the spectral theory (see, e.g. Lemma 9.25, [8]) implies that there is a positive function $w$ such that

$$
L w+\mu_{1} w=0,
$$

that is,

$$
\Delta w+\frac{1}{2}\langle x, \nabla w\rangle+|A|^{2} w-\frac{1}{2} w+\mu_{1} w=0
$$

Then

$$
\begin{aligned}
\mathscr{L} \log w & =\frac{\Delta w+\frac{1}{2}\langle x, \nabla w\rangle}{w}-\frac{|\nabla w|^{2}}{w^{2}} \\
& =-|A|^{2}+\frac{1}{2}-\mu_{1}-|\nabla \log w|^{2}
\end{aligned}
$$

Take $\phi \in C_{0}^{\infty}(\Sigma)$.

$$
\int_{\Sigma} \phi^{2}(\mathscr{L} \log w) e^{\frac{|x|^{2}}{4}} d \sigma=\int_{\Sigma}\left(-|A|^{2}+\left(\frac{1}{2}-\mu_{1}\right)-|\nabla \log w|^{2}\right) \phi^{2} e^{\frac{|x|^{2}}{4}} d \sigma
$$

$$
\begin{aligned}
\int_{\Sigma}\left(|A|^{2}+|\nabla \log w|^{2}\right) \phi^{2} e^{\frac{|x|^{2}}{4}} d \sigma & =\int_{\Sigma}\left(\frac{1}{2}-\mu_{1}\right) \phi^{2} e^{\frac{|x|^{2}}{4}} d \sigma-\int_{\Sigma} \phi^{2}(\mathscr{L} \log w) e^{\frac{|x|^{2}}{4}} d \sigma \\
& =\int_{\Sigma}\left(\frac{1}{2}-\mu_{1}\right) \phi^{2} e^{\frac{|x|^{2}}{4}} d \sigma+\int_{\Sigma}\left\langle\nabla \phi^{2}, \nabla \log w\right\rangle e^{\frac{|x|^{2}}{4}} d \sigma
\end{aligned}
$$

By

$$
\int_{\Sigma}\left\langle\nabla \phi^{2}, \nabla \log w\right\rangle e^{\frac{|x|^{2}}{4}} d \sigma \leq \int_{\Sigma}|\nabla \phi|^{2} e^{\frac{|x|^{2}}{4}} d \sigma+\int_{\Sigma}|\nabla \log w|^{2} \phi^{2} e^{\frac{|x|^{2}}{4}} d \sigma,
$$

(5.3) implies that

$$
\int_{\Sigma}|A|^{2} \phi^{2} e^{\frac{|x|^{2}}{4}} d \sigma \leq \int_{\Sigma}\left(\frac{1}{2}-\mu_{1}\right) \phi^{2} e^{\frac{|x|^{2}}{4}} d \sigma+\int_{\Sigma}|\nabla \phi|^{2} e^{\frac{|x|^{2}}{4}} d \sigma .
$$


Since the spectrum of $\mathscr{L}$ is discrete, there is the first eigenfunction $u>0$ associated to $\lambda_{1}$ for the operator $\mathscr{L}$ such that $u \in W^{1,2}(\Sigma)$ and

$$
\mathscr{L} u+\lambda_{1} u=0 .
$$

Choose $\phi=\varphi_{j} u$, where $\varphi_{j}$ are the non-negative cut-off functions satisfying that $\varphi_{j}$ is 1 on $B_{j},|\nabla \varphi| \leq 1$ on $B_{j+1} \backslash B_{j}$, and $\varphi=0$ on $\Sigma \backslash B_{j+1}$. Substitute $\phi$ in (5.4):

$$
\int_{\Sigma}|A|^{2} \varphi_{i}^{2} u^{2} e^{\frac{|x|^{2}}{4}} d \sigma \leq \int_{\Sigma}\left(\frac{1}{2}-\mu_{1}\right) \varphi_{j}^{2} u^{2} e^{\frac{|x|^{2}}{4}} d \sigma+\int_{\Sigma}\left|\nabla\left(\varphi_{j} u\right)\right|^{2} e^{\frac{|x|^{2}}{4}} d \sigma
$$

Similar to the proof of Theorem 1.4, letting $j \rightarrow \infty$ in (5.5), the monotone convergence theorem implies that

$$
\begin{aligned}
\int_{\Sigma}|A|^{2} u^{2} e^{\frac{|x|^{2}}{4}} d \sigma & \leq \int_{\Sigma}\left(\frac{1}{2}-\mu_{1}\right) u^{2} e^{\frac{|x|^{2}}{4}} d \sigma+\int_{\Sigma}|\nabla u|^{2} e^{\frac{|x|^{2}}{4}} d \sigma \\
& =\int_{\Sigma}\left(\frac{1}{2}-\mu_{1}+\lambda_{1}\right) u^{2} e^{\frac{|x|^{2}}{4}} d \sigma
\end{aligned}
$$

Hence

that is, (1.9) holds:

$$
\frac{1}{2}-\mu_{1}+\lambda_{1} \geq 0
$$

$$
\mu_{1} \leq \lambda_{1}+\frac{1}{2}
$$

If $\frac{1}{2}+\lambda_{1}-\mu_{1}=0$, then $|A| \equiv 0$ on $\Sigma$. Thus $H \equiv 0$, and $\Sigma$ is the hyperplane through the origin. Reciprocally for the $\mathbb{R}^{n}$ through the origin, $L=\mathscr{L}-\frac{1}{2}$. Hence

$$
\mu_{1}=\lambda_{1}+\frac{1}{2}=\frac{n+1}{2}
$$

The rest is to prove $\mu_{1} \geq 1$ in (1.10) in the case that $\Sigma$ is mean convex. It is known that $H \equiv 0$ or $H>0$. If $H \equiv 0$, the conclusion obviously holds. If $H>0$, the Simons' type equation (3.26) states $H$ is a positive solution of

$$
L H+H=\mathscr{L} H+\left(|A|^{2}+\frac{1}{2}\right) H=0 .
$$

This fact implies implies that $\mu_{1} \geq \frac{1}{2}$ (see, e.g., [18, [14]).

\section{REFERENCES}

[1] S. Angenent, T. Ilmanen, and D. L. Chopp, A computed example of nonuniqueness of mean curvature flow in $\mathbb{R}^{3}$, Comm. Partial Differential Equations 20 (1995), 19371958.

[2] Xu Cheng, Tito Mejia, and Detang Zhou, Simons-type equation for $f$-minimal hypersurfaces and applications, J. Geom. Anal. 25 (2015), no. 4, 2667-2686. MR3427142

[3] Stability and compactness for complete $f$-minimal surfaces, Trans. Amer. Math. Soc. 367 (2015), no. 6, 4041-4059, DOI 10.1090/S0002-9947-2015-06207-2. MR3324919

[4] _ Eigenvalue estimate and compactness for closed $f$-minimal surfaces, Pacific J. Math. 271 (2014), no. 2, 347-367, DOI 10.2140/pjm.2014.271.347. MR3267533 
[5] Xu Cheng and Detang Zhou, Stability properties and gap theorem for complete fminimal hypersurfaces, Bull. Braz. Math. Soc. (N.S.) 46 (2015), no. 2, 251-274, DOI 10.1007/s00574-015-0092-z. MR3448944

[6] - Volume estimate about shrinkers, Proc. Amer. Math. Soc. 141 (2013), no. 2, 687-696, DOI 10.1090/S0002-9939-2012-11922-7. MR2996973

[7] _ Eigenvalues of the drifted Laplacian on complete metric measure spaces, Communications in Contemporary Mathematics, DOI 10.1142/S0219199716500012.

[8] Tobias H. Colding and William P. Minicozzi II, Generic mean curvature flow I: generic singularities, Ann. of Math. (2) 175 (2012), no. 2, 755-833, DOI 10.4007/annals.2012.175.2.7. MR2993752

[9] , Smooth Compactness of self-shrinkers, Comment. Math. Helv. 87 (2012), 463-475, DOI 10.4171/CMH/260.

[10] Qi Ding, Minimal cones and self-expanding solutions for mean curvature flows, arXiv:1503.02612 [math.DG] 9 Mar 2015.

[11] Qi Ding and Y.L. Xin, Volume growth eigenvalue and compactness for self-shrinkers, Asian J. Math. 17 (2013), no. 3, 443-456.

[12] Klaus Ecker and Gerhard Huisken, Mean curvature evolution of entire graphs, Ann. of Math. 2nd Ser. 130 (1989), no. 3, 453-471.

[13] Frederick Tsz-Ho Fong and Peter McGrath, Rotational Symmetry of Asymptotically Conical Mean Curvature Flow Self-Expanders, arXiv:1609.02105v1 [math.DG] 7 Sep 2016.

[14] Doris Fischer-Colbrie and Richard Schoen, The structure of complete stable surfaces in 3-manifolds of nonnegative scalar curvature, Comm. Pure Appl. Math. 33 (1980), no. 2, 199-211.

[15] Alexander Grigoryan, Heat Kernel and Analysis on Manifolds, American Mathematical Soc., 2009 (English).

[16] Gerhard Huisken, Local and global behaviour of hypersurfaces moving by mean curvature. Differential geometry: partial differential equations on manifolds (Los Angeles, CA, 1990), Proc. Sympos. Pure Math., 54, Part 1, Amer. Math. Soc., Providence, RI, (1993), 175-191.

[17] T Ilmanen, Lectures on Mean Curvature Flow and Related Equations (Trieste Notes), (1995).

[18] Peter Li and Jiaping Wang, Weighted Poincaré inequality and rigidity of complete manifolds, Ann. Sci. École Norm. Sup. (4) 39 (2006), no. 6, 921-982, DOI 10.1016/j.ansens.2006.11.001 (English, with English and French summaries). MR2316978

[19] Michael Reed and Barry Simon, Methods of modern mathematical physics. IV. Analysis of operators, Academic Press [Harcourt Brace Jovanovich, Publishers], New YorkLondon, 1978. MR0493421

[20] Nikolaos Stavrou, Selfsimilar solutions to the mean curvature flow, J. reine angew. Math. 499 (1998), 189-198.

[21] Ditter Tasayco and Detang Zhou, Uniqueness of grim hyperplanes for mean curvature flows, Arch. Math. (Basel) 109 (2017), no. 2, 191-200, DOI 10.1007/s00013-017-10579. MR3673637

Instituto de Matemática e Estatística, Universidade Federal Fluminense, Niterói, RJ 24020, BRAZIL 